\title{
Object Wide Scope and Semantic Trees ${ }^{1}$ \\ Dorit Ben-Shalom UCLA
}

\section{$1 \quad$ Introduction}

Contrary to what is often assumed, object wide scope (OWS)readings are in fact severely restricted. Liu (1990) conducted the first systematic study of the scope readings of transitive sentences involving a wide variety of quantified NPs. The data in (1) are illustrative of the restrictions she found: 2

(1) a. Two referees read every abstract

'for every abstract there are two referees that read it'

b. Two referees read three abstracts

? 'there are three abstracts and each of them was read by two (possibly different) referees'

c. Two referees read at least three abstracts

?? 'there are at least three abstracts such that each of them was read by two (possibly different) referees'

d. Two referees read exactly three abstracts

* 'there are exactly three abstracts such that each of them was read by two (possibly different) referees'

e. Two referees read more than three abstracts

* 'there are more than three abstracts such that each of them was read by two (possibly different) referees'

f. Two referees read fewer than three abstracts

* 'there are fewer than three abstracts such that each of them was read by two (possibly different) referees'

g. Two referees read no abstracts

* 'there are no abstracts such that each of them was read by two (possibly different) referees'

(1a) is an example of NPs in which the determiner is a distributive universal like every or each. (1b) is an example of NPs whose determiner is a

\footnotetext{
${ }^{1}$ This research was partially supported by NSF grant DBS 9222501. Filippo Beghelli, Ed Keenan, Murat Kural, Friederike Moltmann and Ed Stabler made helpful comments on earlier drafts. The paper would not have been written without Anna Szabolcsi's support and advice. All errors are mine.

${ }^{2}$ We follow Liu in restricting ourselves to non-partitive NPs. While no account of OWS is complete without considering partitives, the judgements concerning their scope taking abilities are delicate and more empirical research is needed before any theoretical conclusions can be reached.
}

Q 1993 by Dorit Ben-Shalom

Utpal Lahiri and Adam Wyner (eds.), SALT III, 19-37, Ithaca, N.Y.: Cornell University. 
numeral: $a$, some or a number like three. (1c)-(1f) are examples of NPs whose determiner is a modified numeral: more than three, exactly three, fewer than three, etc. (1g) is an NP whose determiner is no. Crucially, the readings that are missing in (1) are not incoherent. For example, the missing OWS reading of Two referees read at least three abstracts is the preferred reading of the corresponding passive and negative fronting with subject-aux inversion in (2a) and (2b) (from Szabolcsi and Zwarts(1992)).

(2) a. Fewer than three abstracts were read by two referees

b. Fewer than three abstracts did two referees read

There are two obvious questions to be answered now. First, what is the positive property that characterizes noun phrases that are able to take OWS? And second, whatever this property turns out to be, what is it about OWS that requires the presence of this property? In this paper we suggest that the answers derive from the following specific properties of the syntax-semantics interface:

(3) a. An OWS reading is available only if the object NP is interpreted as a principal filter.

b. The distinctive syntactic property of an OWS reading is that it involves inverse scope: the scope order does not match the order of argument positions (which is roughly the S-structure c-command order of the arguments).

c. Readings involving inverse scope may be derived in either of two ways: (i) Using unary quantifiers, which are applied in an order that reverses their lexical order, or (ii) Using an n-ary quantifier, which applies to the denotation of the verb to yield the denotation of the sentence in a single step.

d. It will be argued that (i), the application of unary quantifiers in a non-lexical order is only possible if lexical argument order is clearly overridden by a syntactic process, e.g. surface movement. This restriction will be motivated empirically, with reference to contrasts like those between (1f) and (2b), as well as theoretically, with reference to an algebraic approach to generalized quantifiers which is called semantic trees.

e. It follows that only (ii), the $n$-ary quantifier option, is available for sentences of type (1). N-ary quantifiers are in general not run-of-the-mill compositional entities. It is therefore not surprising to find that only simple special cases are actually attested in natural language.

f. We show that the OWS reading can be calculated by a particular, relatively simple $n$-ary quantifier just in case the object 
denotes a principal filter. This is the explanation of the observation in (3a).

An alternative proposal to account for Liu's generalizations has been made independently in Beghelli (1993) within the framework of Government and Binding Theory. Roughly, Beghelli assumes that only distributive universals undergo $Q R$. Unmodified indefinites acquire virtual scope via existential closure. Modified indefinites are quantifiers but neither QR nor existential closure is a vailable to them, hence they take scope in situ. Beghelli's predictions and mine are by and large compatible; a detailed comparison is outside the scope of this paper.

\section{On the calculation of OWS readings}

In this section we provide intuitive motivation for the claim that OWS readings are derived using the binary quantifier $(\mathrm{Q} 2>\mathrm{Q1})$ in (4).

(4) $(Q 2>Q 1)(\lambda x \lambda y[R(x, y)])=$ true iff $\forall a \in A, Q 1(\lambda x[R(x, a)])=$ true,

where $\mathrm{A}$ is a unique set determined by $\mathrm{Q} 2$.

Let (5) be a simple transitive sentence.

(5) NP1 [V NP2]

To say that (5) has an OWS reading is to claim that it can have the meaning in (6), where Q2 is the quantifier expressed by NP2, Q1 is the quantifier expressed by NP1, and $R$ is the relation expressed by V.

(6) $\quad Q 2(\lambda y[Q 1(\lambda x[R(x, y)])])$

Standard scope mechanisms like QR derive the meaning in (6) for any sentence of the form (5) in which NP1 and NP2 are quantified NPs. In particular, they treat NPs like every abstract and fewer than three abstracts exactly alike. They thus derive the reading in (6) for the sentences in both (7a) and (7b).

(7) a. Two referees read every abstract 'for every abstract there are two referees that read it'

b. Two referees read fewer than three abstracts

* 'there are fewer than three abstracts such that each of them was read by two (possibly different) referees'

Using the standard view of compositional semantics, let us assume that (6) actually encodes the way in which the desired meaning is obtained. It says then that (8) is a subpart of the meaning of (6): 
(8) $\quad \lambda y[Q 1(\lambda x[R(x, y)])]$

This amounts to saying that ( $7 \mathrm{a}$ ) is calculated by first forming the set of entities that are read by two referees, and then asking whether this set contains every abstract. Similarly, in the case of (7b), we calculate the set of entities read by two referees and ask whether this set contains fewer than three abstracts.

Now we know that ( $7 a$ ) does have the predicted reading and ( $7 b$ ) does not. This suggests that in reality even the existing reading of $(7 \mathrm{a})$ is not obtained in the way described above. Presumably, it is obtained in a different way that is not available to ( $7 \mathrm{~b})$, hence the contrast.

One alternative way for calculating $(7 a)$ is as follows. Instead of starting with forming the set of things read by two referees, start with the set of abstracts and ask whether every one of them was read by two referees. In this case the set of things that were read by two referees is never constructed. ${ }^{3}$

In the case of $(7 \mathrm{a})$ this latter way of calculating the OWS seems to calculate the right truth conditions. Will this be the case for any choice of the quantifiers Q1 and Q2? Notice that this way of calculating has a semantic prerequisite: the wide scope quantifier Q2 must somehow determine a unique set whose members can be checked in the desired way. Hence the prediction is that this calculation will only be available if the quantifier Q2 provides such a set. In what follows we will prove that the quantifiers that provide the desired set are precisely the principal filters, i.e., we prove that the two ways of calculating are equivalent just in case Q2 is a principal filter. The next step is to ask which NPs can denote principal filters. We argue below that every abstract and three abstracts, for instance, can, while modified numerals like least three abstracts, fewer than three abstracts and exactly three abstracts, cannot. Notice that Liu (1990) found that NPs like every abstract and three abstract are among those that are capable of taking OWS, while NPs like at least three abstracts, fewer than three abstracts and exactly three abstracts are among those that are not. Thus our speculation concerning calculation converges with the empirical data. We will suggest that a natural way to capture the calculation with principal filters is to use a binary quantifier like (4). This explains why every abstract and three abstracts take OWS but at least three abstracts, fewer than three abstracts and exactly three abstracts do not: only the former satisfy the semantic prerequisite for the use of (4). We may note here that Liu characterized the OWS class with a combination of semantic and morphological properties: NPs that denote upward monotonic quantifiers and do not contain a "modifier" (at least, more than, etc.). Our proposal implies that such a heterogeneous characterization is not necessary: OWS-takers can be characterized in semantic terms. 4 In sections 3 and

\footnotetext{
${ }^{3}$ Note the close parallel with Szabolesi and Zwarts (1992)'s distinction between 'compute' and 'look-up' procedures (Section 5.1).

${ }^{4}$ The principal filterhood requirement should be understood as a necessary but not as a
} 
4 we elaborate on the semantic properties of the binary quantifier Q1>Q2. In section 5 we take up the discussion of the syntax-semantics interface that makes it natural to employ specifically a binary quantifier here. Section 6 places these considerations in the context of semantic trees.

\section{Object wide scope and principal filters}

\subsection{Definitions and notational conventions}

We are using the term 'quantifiers' for semantic objects, as in Barwise and Cooper (1981). A unary quantifier is a set of sets. An n-ary quantifier is a set of n-ary relations. A binary quantifier is a set of binary relations. The corresponding syntactic notions involve the number of different variables bound by the quantifier: one, more than one and two, respectively. To avoid confusion, please note the following notational conventions.

As is usual, we sometimes think of sets as their characteristic functions. In particular, we sometimes think of a unary quantifier as a function from sets to the truth values 'true' and 'false'. So we use expressions like (9a) interchangeably with expressions like (9b):

(9) a. $\quad\{1,2,3\} \in Q$

b. $Q(\{1,2,3\})=$ true

Similarly, we sometimes think of a binary quantifier as function from binary relations to the truth values 'true' and 'false'. We also use lambda notation to represent some sets and binary relations. So a binary relation is written as either $(10 \mathrm{a})$ or $(10 \mathrm{~b})$.

(10) a. $R$

b. $\quad \lambda x \lambda y[R(x, y)]$

An expression like (11b) in which $Q$ is a binary quantifier is therefore just another way of saying (11a).

$$
\begin{array}{ll}
\text { a. } & R \in Q \\
\text { b. } & Q(\lambda x \lambda y[R(x, y)])=\text { true }
\end{array}
$$

Finally, we will consistently use Q2 for the denotation of the object and Q1 for the denotation of the subject.

sufficient condition for OWS taking. Another semantic property that is likely to play a role when partitives and definite NPs are considered is distributivity, as (ii) below has an OWS reading while (i) does not.

(i) Two referees read the abstracts

(ii) Two referees read each of the abstracts 


\subsection{The binary quantifier Q2>Q1}

We start by making two standard assumptions. First, an OWS reading expresses the meaning in $(12)(=6)$.

$$
Q 2(\lambda y[Q 1(\lambda x[R(x, y)])])
$$

Second, the existence of an OWS for a sentence does not depend on the value of $\mathrm{R}$, e.g, whether $T$ wo referees read every abstract has an OWS reading does not depend on who read what. In other words, whatever is the mechanism that derives OWS readings, it has to derive the reading in (12) for every binary relation $R$. In view of the intuitive consideration in section 2 , we are also making the specific assumption that OWS readings are derived using the binary quantifier Q2 $>$ Q1 in $(13)(=4)$.

(13) $(Q 2>Q 1)(\lambda x \lambda y[R(x, y)])=$ true

iff $\forall a \in A, Q 1(\lambda x[R(x, a)])=$ true,

where $\mathrm{A}$ is a unique set determined by $\mathrm{Q} 2$.

In section 3.3 we formally address the question for which quantifiers Q1 and $Q 2$ the readings $(Q 2>Q 1)(\lambda x \lambda y[R(x, y)])$ and $Q 2(\lambda y[Q 1(\lambda x[R(x, y)])])$ are equivalent for every binary relation $R$, and prove that this equivalence holds just in case Q2 is a principal filter.

3.3 The formal role of principal filters in Q2>Q1

Let $\mathrm{Q1,Q2}$ be unary quantifiers over a universe $\mathrm{E}$.

Lemma If $Q 1$ is non-trivial ${ }^{5}$ then there is a set $A$ such that (i) and (ii) are equivalent for all binary relations $\mathrm{R}$, iff $\mathrm{Q} 2$ is a principal filter.

(i) $\forall a \in A, Q 1(\lambda x[R(x, a)])=$ true

(ii) $Q 2(\lambda y[Q 1(\lambda x[R(x, y)])])=$ true

Proof:

Since $\mathrm{Q} 1$ is non trivial, $\lambda y[Q 1(\lambda x[R(x, y)])]$ can be any property $\mathrm{P}$, i.e., for every property $\mathrm{P}$ there is a binary relation $\mathrm{R}$ such that $\lambda y[Q 1(\lambda x[R(x, y)])]$ $=P$. Therefore it is enough to prove that there is a set $A$ such that (i') and (ii') are equivalent for every property $\mathrm{P}$, iff $\mathrm{Q} 2$ is a principal filter.

(i') $\forall a \in A, P(a)=$ true

(ii') $Q 2(\lambda y[P(y)])=$ true

Thinking about quantifiers now as sets of sets, this amounts to proving that there is a set A such that ( $\left.\mathrm{i}^{\eta}\right)$ and (ii”) are equivalent for every set $\mathrm{X}$, iff Q2 is a principal filter.

\footnotetext{
${ }^{5}$ i.e., $\mathrm{Ql}$ is not always true or always false.
} 
(i”) $A \subseteq X$

(ii") $X \in Q 2$

And this is true by definition, as principal filters are defined as in (14).

(14) A quantifier Q2 is a principal filter iff $\exists A \subseteq E$ such that $\forall X \subseteq E, A \subseteq X$ iff $X \in Q 2 \quad$ 口

The set A in (14) is called the generating set of the principal filter Q2, and Q2 is said to be generated by A. Formally, then, a quantifier is a principal filter iff it is generated by some set $A$.

\subsection{What noun phrases denote principal filters?}

A distributive universal like every abstract is interpreted as a principal filter generated by the set denoted by its common noun. For example, every abstract is interpreted by the principal filter EVERY ABSTRACT generated by the set ABSTRACT: a set X is a set in EVERY ABSTRACT iff it contains all abstracts. ${ }^{6}$ Bare numerals like three abstracts are in general not interpreted as principal filters, as we will see below. There is, however, one reading of three abstracts which we will claim is a principal filter, namely the referential reading of Fodor and Sag (1982).

When interpreted referentially, an NP like three abstracts is interpreted by a quantifier like THREE ABSTRACTS with the following meaning: there are three concrete (but arbitrarily chosen) abstracts such that a set $\mathrm{X}$ is a set of THREE ABSTRACTS iff it contains those three abstracts. So THREE ABSTRACTS is generated by a set of three (arbitrarily chosen) abstracts. ${ }^{7}$

Under the non-referential reading, bare numerals like three abstracts are interpreted like the modified numeral at least three abstracts, which is interpreted by the quantifier AT LEAST THREE ABSTRACTS. It is easy to show that AT LEAST THREE ABSTRACTS is not a principal filter, because is not generated by any set A: If $A$ contains all abstracts, then in any world with more than three abstracts, there is a set $\mathrm{X}$ such that $\mathrm{X}$ is in AT LEAST THREE ABSTRACTS, but $A$ is not a subset of $X$. In fact, any set of exactly three abstracts will do. If $A$ does not contain all abstracts, then there is some abstract $a_{1}$ that is not in $A$. Since the world contains at least three abstracts we can form a set $X$ such that $X$ is in AT LEAST THREE ABSTRACTS but $A$ is not a subset of $X$ : for example $X$ can be any set of three abstracts containing $a_{1}$.

\footnotetext{
${ }^{6} \mathrm{NPs}$ can be accidentally interpreted as principal filters simply because the universe $\mathrm{E}$ is too small. For example, EXACTLY THREE ABSTRACTS is a filter in a world with only three abstracts. To deal with these cases it is possible to require that there is some model $M$ such the NP is interpreted as a principal filter in $M$ and all of its extensions.

${ }^{7}$ Thus, the abstracts are concrete but not pragmatically specific in any way. It is not required that the speaker or anyone else knows which abstracts they are.
} 
Similarly, a monotone decreasing quantifier like FEWER THAN THREE ABSTRACTS is never a principal filter in any world with at least three abstracts. Again, we can see that FEWER THAN THREE ABSTRACTS cannot be generated by any set $A$. If $A$ is not empty, then the empty set is a set $X$ such that $X$ is in FEWER THAN THREE ABSTRACTS but $A$ is not a subset of $X$. If $A$ is the empty set, then any set $X$ with at least three abstracts is a superset of the empty set but is not in FEWER THAN THREE ABSTRACTS.

\subsection{An example}

Suppose the world is as in (15).

(15) John, Bill and Mary are the only referees 1,2,3,4 are the only abstracts John read abstracts 1,2 and 4, Bill read abstracts 1 and 3, Mary read abstracts 2,3 and 4 , and no one else read anything else.

For readability, we represent relations graphically by tres. So the READ relation in (15) is represented by the tree in (16).

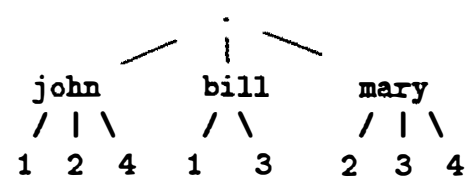

The OWS reading of Two referees read every abstract can be calculated by the binary quantifier EVERY ABSTRACT>TWO REFEREES where A is ABSTRACT, the generating set of EVERY ABSTRACT. For example, if the READ relation is as in (15), then (EVERY ABSTRACT>TWO REFEREES)(READ) is true since every member of the set ABSTRACT was read by two referees.

The OWS reading of Two referees read fewer than three abstracts on the other hand, cannot be calculated by the binary quantifier FEWER THAN THREE ABSTRACTS > TWO REFEREES no matter what $A$ is. If $A$ contains fewer than three abstracts then (FEWER THAN THREE ABSTRACTS $>$ TWO REFEREES)(READ) is true if the READ relation is as in (15), since every member of A was read by two referees. But the OWS reading of Two referees read fewer than three abstracts is false in this case, because four abstracts were read by two referees. If $A$ contains three abstracts or more, then (FEWER THAN THREE ABSTRACTS > TWO REFEREES)(READ) is false if fewer than three abstracts were read by two referees, for example if the READ relation is as represented by the tree in (17). 


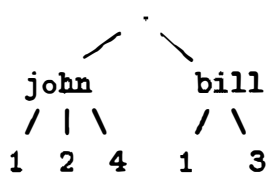

\section{Empirical alternatives and extensions}

\subsection{Alternative definitions of Q2>Q1}

We defined Q2>Q1 as in (13) above to account for the empirical generalization that NPs that take OWS can be interpreted as principal filters. If we had found, instead, that NPs that cannot be interpreted as principal filters take OWS too, we would have followed a similar reasoning using a different definition of Q2>Q1. For example, suppose NPs like at least three abstracts that are interpreted as monotone increasing quantifiers, but not as principal filters, had taken OWS. Then Q2>Q1 could be defined by (18) instead of (13) above. $^{8}$

$(Q 2>Q 1)(\lambda x \lambda y[R(x, y)])=$ true

iff $\exists A \in Q 2$ such that $\forall a \in A, Q 1(\lambda x[R(x, a)])=$ true

This definition predicts that NPs that can take OWS can be interpreted as monotone increasing quantifiers, because if Q2>Q1 is defined as in (18), then $(Q 2>Q 1)(\lambda x \lambda y[R(x, y)])$ expresses an OWS reading just in case Q2 is monotone increasing. ${ }^{9}$ It is worth noting, however, that calculating with the binary quantifier in (18) is less simple than calculating with the binary quantifier in (13): (13) involves a fixed set $A$ for each quantifier $Q 2$, whereas (18) can in principle involve all the sets in Q2.

It is even possible to modify $Q 2>Q 1$ in such a way that it calculates OWS readings for object NPs like FEWER THAN THREE ABSTRACTS or EXACTLY THREE ABSTRACTS. But the resulting binary quantifier is significantly more complex than even (18), because these object NPs are interpreted as quantifiers that are not monotone increasing: If Q2 is not monotone increasing then the set $\mathrm{A}$ has to be maximal in some way with respect to

\footnotetext{
${ }^{8}$ The set $A$ in (18) is required to be a witness of $Q 2$. A more intuitive but equivalent definition would require it to be a witness* of Q2, namely a witness set that is a subset of every set that Q2 lives on. For the definitions of 'witness' and 'live on' see Barwise and Cooper (1981).

${ }^{9}$ This formal claim follows from the definition of monotone increasing quantifiers in (i) by an argument similar to the one in Section $\mathbf{3 . 3}$ above.
}

(i) A quantifier Q2 is monotone increasing

iff $\forall X \subseteq E,(\exists A \in Q 2$ such that $A \subseteq X)$ iff $X \in Q 2$ 
$\lambda y[Q 1(\lambda x[R(x, y)])]$. For example, if there is a set $\mathrm{A}$ of fewer than three abstracts that were read by two referees each, it does not mean that fewer than three abstracts were read by two referees.

\subsection{Possible extensions: three-place predicates}

We conclude this section with a brief informal discussion of possible extensions of the analysis to inverse scope with thre-place predicates.

A reading like (19), in which an indirect object takes scope over a direct object, can be derived by practically the same binary quantifier as EVERY STUDENT > TWO QUESTIONS.

(19) At least four teachers assigned two questions to every student

'at least four teachers are such that they assigned every student two (possibly different) questions'

A reading like (20), in which an indirect object takes scope over both the subject and the direct object can be derived by a ternary quantifier, i.e., a set of ternary relations. This quantifier will be very similar in structure to Q2>Q1, but the relevant $Q 1$ will be a binary quantifier itself.

(20) At least four teachers assigned two questions to every student 'every student is such that at least four teachers assigned two (possibly different) questions to him'

A more drastic extension is needed to derive readings like (21), if they exist. The judgements turn out to be very delicate, especially if one is careful about distinguishing this reading from the relatively close readings in footnote 10. 1011

(21) At least four teachers assigned two questions to three students 'at least four teachers are such that they assigned each of three (possibly different) students two (possibly different) questions'

\footnotetext{
${ }^{10}$ The reading in (21) is different than the branching reading (under which different teachers may assign different questions, but each teacher assigned the same two questions to " his" three students). It is also different than the reading in which the two of two questions is focused (under which each teacher assigned exactly two (possibly different) questions to each of the students he assigned questions to).

${ }^{11}$ It is easier to get a reading superficially similar to (21) for the sentence in (i). But this is arguably the "incorporation" reading 'at least four teachers are such that they assigned questions to three students'
}

(i) At least four teachers assigned a question to three students 
To get the reading in (21) it would be necessary to assume that the crucial set $\mathrm{A}$ in the definition of $(Q 2>Q 1)(\lambda y \lambda z[R(x, y, z)])$ can depend on $x$. That this kind of extension might be needed anyway might be indicated by readings like (22), in which an indirect object bound by the subject is taking scope over a direct object. ${ }^{12}$

(22) At least four teachers assigned two questions to each of their students 'at least four teachers are such that they assigned each of their students two (possibly different) questions'

\section{Object wide scope and binary quantifiers}

We are arguing that OWS relies on a binary quantifier Q2 $>$ Q1 that is defined only when Q2 denotes a principal filter. Until now we have motivated the semantic aspects of this claim. The next thing to be explained is why we need specifically a binary quantifier for this purpose. This question pertains to the syntax-semantics interface.

\subsection{On productive rebracketing}

Let us recall from section 2 that an OWS reading in the general case is expressed by (23) (=6):

$$
Q 2(\lambda y[Q 1(\lambda x[R(x, y)])])
$$

As was pointed out there, this amounts to saying that (24), which combines the meanings of the subject and the verb, to the exclusion of the object, is a subpart of the meaning of the sentence:

$$
\lambda y[Q 1(\lambda x[R(x, y)])])
$$

Under the assumption of compositionality, this entails that there is a syntactic constituent of the sentence that carries precisely this meaning. In grammars like Montague (1974) or May (1977), such a constituent is readily available. It is produced by quantifying in (25a) or, equivalently, by Quantifier Raising (25b):

a.

$$
\text { Two referees read fewer than three abstracts }
$$

${ }^{12}$ That specificity does not exclude referential dependency is indicated by sentences like
(i), which has a natural reading under which different boys are interested in different girls.

(i) Every boy expressed an interest in a certain girl. 
b. [s fewer than three abstracts ${ }_{i}\left[s\right.$ two referees $j\left[\left[s t_{j}\right.\right.$ read $\left.\left.t_{i}\right]\right]$

Similarly, a flexible categorial grammar might achieve a comparable rebracketing of the NP.VP structure by commuting the arguments of the verb, or by composing the subject with the verb. We have seen that these syntactic processes overgenerate: they derive an OWS reading for any transitive sentence, including Two referees read fewer than three abstracts, which does not have any. On the other hand, we have also seen that the missing OWS readings reappear, for instance, when passivization or object fronting applies:

(26) a. Fewer than three abstracts were read by two referees

b. Fewer than three abstracts did two referees read

From these we conclude that the grammar should not have productive, unconstrained syntactic devices that override the lexical hierarchy of arguments. Such devices should only be available if there is independent justification for their operation. Surface movement is one example. This entails that if the sentence exhibits no signs of syntactic rebracketing, an OWS reading can only be obtained in a special, non-productive manner. In 5.2 we show that under certain assumptions about semantic interpretation, this amounts to saying that the OWS reading is derived by a binary quantifier. In section 6 we go on to show that the assumptions of 5.2 find their natural place in the context of semantic trees.

\subsection{Object wide scope as binary quantification}

We make the following assumptions concerning the semantic interpretation of simple transitive sentences. First, the semantic interpretation of a transitive sentence without any surface movement consists of successive applications of the denotations of the NPs to the denotation of the verb. Second, NPs are interpreted as quantifiers that "saturate" the last ${ }^{13}$ argument place of the predicate they apply to. Formally, the last assumption amounts to interpreting NPs by particular extensions (projections, lifts) of unary generalized quantifiers. The value of such a quantifier $\mathbf{Q}$ at an $n$-ary relation $\lambda x_{1}, \ldots \lambda x_{n}\left[R\left(x_{1}, \ldots, x_{n}\right)\right]$ is defined in (27), adapted from Keenan (1992).

$$
\begin{aligned}
& Q\left(\lambda x_{1}, \ldots \lambda x_{n}\left[R\left(x_{1}, \ldots, x_{n}\right)\right]\right)=\operatorname{def} \\
& \lambda x_{1}, \ldots, \lambda x_{n-1}\left[Q\left(\lambda x_{n}\left[R\left(x_{1}, \ldots, x_{n-1}, x_{n}\right)\right]\right)\right]
\end{aligned}
$$

One consequence of our assumptions is that a subject wide scope reading can always be derived in a unary way, i.e., by successively applying the denotations of the NPs to the denotation of the verb. Formally, this is because

\footnotetext{
${ }^{13}$ 'Last' refers to the left to right order of the argument places. We are assuming that the lexical entry of a transitive verb $\mathrm{V}$ is $\mathrm{V}$ (agent,theme), where 'agent' and 'theme' are used, as usual, simply as cover terms for the actual thematic roles.
} 
a subject wide scope reading is of the form $Q 1(\lambda x[Q 2(\lambda y[R(x, y)])])$, which can be derived in accordance with (27) by first applying the denotation of the object to the denotation of the verb, and then applying the denotation of the subject to the result.

It also follows from these assumptions that an OWS reading cannot be derived in this particular way. Formally, this is because an OWS reading is of the form $Q 2(\lambda y[Q 1(\lambda x[R(x, y)])])$. And $\lambda y[Q 1(\lambda x[R(x, y)]]$ cannot be derived in accordance with (27) by applying Q1, the denotation of the subject NP to the denotation of the transitive verb. Thus, the only way to derive an OWS reading in accordance with the assumptions above is to derive it in a binary way, i.e., by interpreting the subject and the object together as one binary quantifier. Formally, this amounts to interpreting the pair consisting of the subject NP and the object NP as a binary quantifier $Q$, and deriving the OWS reading as $Q(\lambda x \lambda y[R(x, y)])$.

If, on the other hand, the object is syntactically fronted as in (28) and a constituent with the interpretation $\lambda y[Q 1(\lambda x[R(x, y)])]$ is provided, the OWS reading does not require binary quantification:

(28) Fewer than three abstracts did two referees read 'there are fewer than three abstracts such that each of them was read by two (possibly different) referees'

\section{Semantic Trees}

Untill now we have used trees simply as graphic representations of relations. While they do serve such an illustrative purpose, in Ben-Shalom (1992, in progress) they are also entities of a theory that treats NP-denotations as operations on n-ary relations. Algebraically, classes of such operations are characterized by invariance conditions on a set of pairs consisting of a k-tuple and a $\mathrm{k}+\mathrm{l}$-ary relation. One advantage of this approach is that it allows one to talk about generalized quantifiers as well as argument and predicate anaphors, superlatives, etc. in a uniform fashion, as special cases of arity reducing operations. In this paper we will exploit the fact that this theory has an intuitive representation in terms of graphic trees, and so the intuitions behind it can be conveyed without recourse to algebraic devices. Specifically, in 6.1 we give an interpretation of the definition in (27) as tree operations on subtrees of height one. This allows one to see why subject wide scope should be productive and object wide scope should not. In 6.2 we use tree operations to give a simple proof of the unreducibility of the quantifier EVERY ABSTRACT $>$ TWO REFEREES.

\subsection{Generalized quantifiers as tree operations}

The extended generalized quantifiers as defined in (27) correspond to tree operations that operate on subtrees of height one, one at a time. Suppose the world is as in (15) above, repeated as (29): 
(29) John, Bill and Mary are the only referees. 1,2,3,4 are the only abstracts. The READ relation is as represented by the tree in (30)

(30)

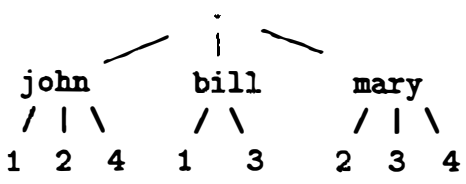

The value of THREE ABSTRACTS(READ) can be calculated as in (31), where the operation defined by the quantifier THREE ABSTRACTS operates on a subtree of height one, one at a time: If THREE ABSTRACTS is true of the set of objects dominated by the root of the subtree, the subtree is deleted but its root is kept ((31b) and (31d)). If THREE ABSTRACTS is false of the set of objects dominated by the root, both the subtree and its root are deleted $((31 c))$.

(31) a

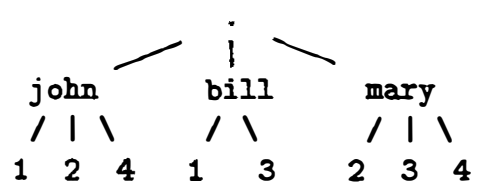

b.

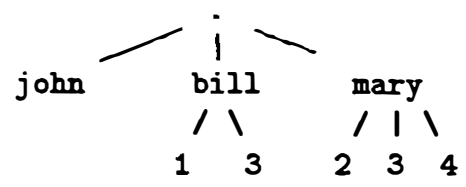

c.

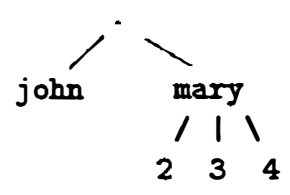

d.

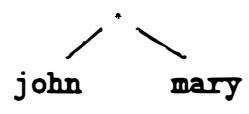

It is easy to see that a subject wide scope reading can always be calculated in such a way that (the operations defined by) the denotations of the NPs operate on subtrees of height one of the semantic tree representing the denotation of the verb: the denotation of the object NP operates on the denotation of the verb as in (31), and the denotation of the subject operates on the resulting tree. For example, the value of the subject wide scope reading 
of Fewer than four referees read three abstracts can be calculated by applying the operation defined by FEWER THAN FOUR REFEREES to the tree representing THREE ABSTRACTS(READ) in (31d) above. Since FEWER THAN FOUR REFEREES is true of the set $\{$ john,mary\} the truth value of this reading in the world we are assuming is 'true'.

It is also easy to see that an OWS reading cannot be calculated in such a way that (the operations defined by) the denotations of the NPs operate on subtrees of height one of the semantic tree representing the denotation of the verb. Rather, it is the binary quantifier derived from the denotations of both the subject and the object that operates on the semantic tree of height two which represents the denotation of the verb. For example, the value of the OWS reading of Two referees read every abstract can be calculated by applying the binary quantifier (EVERY ABSTRACT>TWO REFEREES) to the tree representing the READ relation in (30). Since EVERY ABSTRACT $>$ TWO REFEREES is true of the binary relation represented by (30), the truth value of this reading in the world we are assuming is 'true'.

\subsection{The unreducibility of object wide scope readings}

A binary quantifier $Q$ is called unreducible if there are no unary quantifiers Q3 and Q4 such that (32a) has the same truth value as (32b) for every binary relation $R$.

$$
\begin{array}{ll}
\text { a. } & Q(\lambda x \lambda y[R(x, y)]) \\
\text { b. } & Q 3(\lambda x[Q 4(\lambda y[R(x, y)])])
\end{array}
$$

In this section we use semantic trees to prove that the meaning expressed by the OWS reading of Two referees read every abstract defines an unreducible binary quantifier. That is to say, there are no unary generalized quantifiers Q3 and Q4 such that (33a) has the same truth value as (33b) for every R:

$$
\begin{array}{ll}
\text { a. } & E V E R Y A B S T R A C T(\lambda y[T W O R E F E R E E S(\lambda x[R(x, y)])]) \\
\text { b. } & Q 3(\lambda x[Q 4(\lambda y[R(x, y)])])
\end{array}
$$

We prove this fact by a graphic proof technique that is based on semantic trees. The technique allows for a simplification of the unreducibility proofs in van Benthem (1989) and Keenan (1992). It is formally motivated in BenShalom (1992).

For the purpose of the proof it will be convenient to think of both (33a) and (33b) as defining binary quantifiers. We will call them by the mnemonic names EVERY ABSTRACT-TWO REFEREES and Q3oQ4, respectively. We will think of binary quantifiers as functions from binary relations to truth values, and rewrite (33) as (34).

$$
\text { a. (EVERYABSTRACT - TWO REFEREES })(R)
$$


b. $\quad(Q 3 \circ Q 4)(R)$

Our overall goal is to prove that whatever unary quantifiers Q3 and Q4 are, Q3oQ4 will have a different truth value than EVERY ABSTRACT -TWO REFEREES on at least one binary relation $R$. To prove this we prove that whatever unary quantifier $Q 4$ is, there are two binary relations $R$ and $R$ ' such that Q3oQ4 has the same truth value on both $R$ and $R$ ', whereas EVERY ABSTRACT-TWO REFEREES is true of R and false of R'.

We apply the following graphic reasoning. Suppose Q4 is AT LEAST TWO ABSTRACTS, and that $R$ is the binary relation represented by the tree in (35).

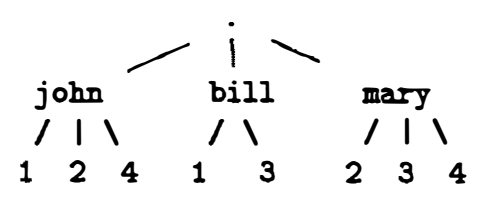

Then whether john is a member of AT LEAST TWO ABSTRACTS $(\lambda y[R(x, y)])$ depends only on the truth value of AT LEAST TWO ABSTRACTS $(\{1,2,4\})$. Therefore (Q3oAT LEAST TWO ABSTRACTS) (R) is the same truth value as (Q3-AT LEAST TWO ABSTRACTS)( $\left.\mathrm{R}^{\prime}\right)$, where R' is the binary relation represented by the tree in (36).

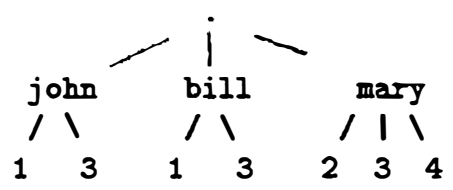

This is so because AT LEAST THREE ABSTRACTS $(\{1,2,4\})=$ AT LEAST THREE ABSTRACTS $(\{1,3\})$, and the only difference between $R$ and $R$ ' is that the subtree in which 'john' dominated ' 1 ' and ' 2 ' and ' 4 ' was replaced by a subtree in which 'john' dominates ' 1 ' and ' 3 '.

Deleting a subtree is just the special case of replacing a subtree with the empty subtree. So suppose Q4 is the unary quantifier FEWER THAN THREE ABSTRACTS, $R$ is the binary relation represented by the tree in (36), and $R^{\prime}$ is the binary relation represented by the tree in (37).

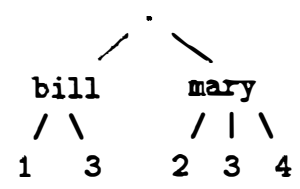


Then (Q3oFEWER THAN THREE ABSTRACTS $)(\mathrm{R})=(\mathrm{Q} 3 \circ$ FEWER THAN THREE ABSTRACTS)(R), because FEWER THAN THREE ABSTRACTS $(1,3)$ $=$ FEWER THAN THREE ABSTRACTS $(\emptyset)$, and the only difference between $R$ and $R$ ' is that the subtree headed by 'john' was deleted. The last fact we need for the proof is that we can assume that Q4 is positive, i.e., Q4 $(\theta)=$ false. Intuitively, this is true because of equivalences like the following: 'Every referee read fewer than three abstracts' is equivalent to 'No referee read at least three abstracts'.

The proof:

We show that there is no unary quantifier Q4 such that (EVERY ABSTRACTTWO REFEREES $)(R)=(Q 3 \circ Q 4)(R)$ for every binary relation $R$.

1. If $Q 4(\{1,2,4\})=$ false, then $(Q 3 \circ Q 4)(R)=(Q 3 \circ Q 4)\left(R^{\prime}\right)$, where $R$ is the binary relation represented by (38a) and $R^{\prime}$ is the binary relation represented by (38b).

(38) a.

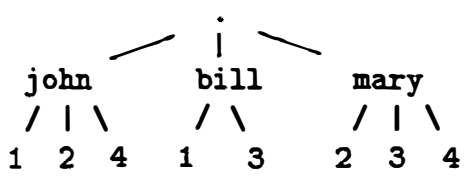

b.

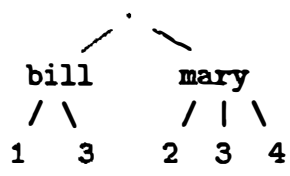

But (EVERY ABSTRACT-TWO REFEREES)(R) = true and (EVERY ABSTRACT - TWO REFEREES)(R') = false, because every abstract was read by two referes in $R$, but abstracts 1,2 and 4 were not read by two referees in R'.

2. Similarly, if $Q 4(\{1,3\})=$ false, then $(Q 3 \circ Q 4)(R)=(Q 3 \circ Q 4)\left(R^{\prime}\right)$, where $R$ is the binary relation represented by $(39 a)$ and $R^{\prime}$ is the binary relation represented by (39b).

a.

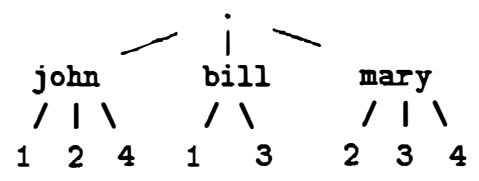

b.

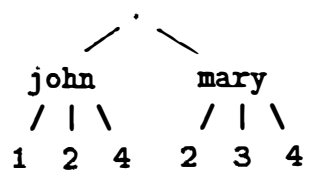


But (EVERY ABSTRACT-TWO REFEREES)(R) = true and (EVERY ABSTRACT- TWO REFEREES)(R') = false, because every abstract was read by two referees in $R$, but abstracts 1 and 3 were not read by two referees in $\mathrm{R}^{\prime}$.

3. The only other option is that $\mathrm{Q} 4(\{1,2,4\})=\mathrm{Q} 4(\{1,3\})=$ true, so $(\mathrm{Q} 3 \circ \mathrm{Q} 4)(\mathrm{R})=(\mathrm{Q} 3 \circ \mathrm{Q} 4)\left(\mathrm{R}^{\prime}\right)$, where $\mathrm{R}$ is the binary relation represented by (40a) and $R^{\prime}$ is the binary relation represented by (40b).

a.

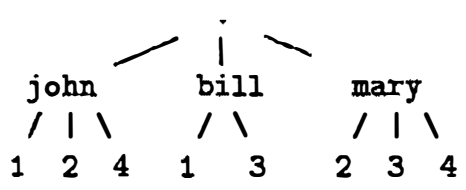

b.

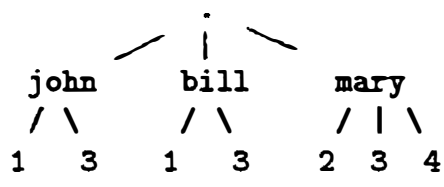

But in this case again, (EVERY ABSTRACT-TWO REFEREES)(R) $=$ true and (EVERY ABSTRACT-TWO REFEREES)(R') = false, because every abstract was read by two referees in $R$, but abstracts 2 and 4 were not read by two referees in R'.

Therefore there is no unary quantifier Q4 such that (EVERY ABSTRACTTWO REFEREES $)(R)=(Q 3 \circ Q 4)(R)$ for every binary relation $R$. Therefore EVERY ABSTRACT-TWO REFEREES is an unreducible binary quantifier.

\section{Conclusion}

In this paper we have argued for the claims in (41):

(41) a. An Object Wide Scope (OWS) reading exists only if the object $\mathrm{NP}$ is interpreted as a principal filter.

b. The claim in (a) follows if an OWS reading is derived by a binary quantifier in which the interpretation of the object NP defines a crucial domain.

c. The claim that OWS readings are derived by binary quantification can be motivated within an algebraic approach to generalized quantifiers which is called semantic trees.

\section{References}

Barwise, J. and R. Cooper (1981) Generalized Quantifiers and and Natural Language in Linguistics and Philosophy 4, 159 - 219. 
Beghelli, F. (1993) A Minimalist Approach to Quantifier Scope Proceedings of NELS 23.

Ben-Shalom, D. (1992) A Tree Characterization of Generalized Quantifier Reducibility UCLA Working Papers in Cognitive Science UCLA-CSRP-927.

Ben-Shalom, D. (in progress) Semantic Trees Ph.D Diss. UCLA.

van Benthem, J. (1989) Polyadic Quantifiers in Linguistics and Philosophy 12, $437-465$.

Fodor, J. and I. Sag (1982) Referential and Quantified Indefinites in Linguistics and Philosophy 5, 355 - 398.

Keenan, E.L. (1992) Beyond the Frege Boundary in Linguistics and Philosophy $15,199-221$.

Liu, F-H. (1990) Scope Dependency in English and Chinese Ph.D Diss. UCLA.

May, R. (1977) The Grammar of Quantification Ph.D Diss. MIT. Reproduced by the Indiana University Linguistics Club.

Montague, R. (1974) Formal Philosophy (ed. R. Thomason), Yale University Press.

Szabolcsi, A. and F. Zwarts (1992) Weak Islands and an Algebraic Semantics for Scope-Taking to appear in Natural Language Semantics.

Dorit Ben-Shalom

Department of Linguistics

UCLA

405 Hilgard Ave.

Los Angeles, CA 90024-1543

dorit@cognet.ucla.edu 